\title{
Analytical Solution for the Boundary Value Problem of Elastic Beams Subjected to Distributed Load
}

\author{
"Mustapha Adewale Usman, Fatai Akangbe Hammed and \\ Debora Oluwatobi Daniel \\ Department of Mathematical Sciences, Olabisi Onabanjo University, \\ Ogun State, Nigeria \\ "Corresponding author: Usman.mustapha@oouagoiwoye.edu.ng
}

Published online: 31 May 2021

To cite this article: Mustapha Adewale Usman, Fatai Akangbe Hammed and Debora Oluwatobi Daniel (2021). Analytical solution for the boundary value problem of elastic beams subjected to distributed load. Journal of Engineering Science, 17(1), 75-93, https://doi.org/10.21315/jes2021.17.1.5.

To link to this article: https://doi.org/10.21315/jes2021.17.1.5

\begin{abstract}
Analytical solution for the boundary value problem (BVP) of elastic beams subjected to distributed load was investigated. Based on the study, dynamic application curves are developed for beam deflection. The partial differential equation of order four were analysed to determine the dynamic response of the elastic beam under consideration and solved analytically. Effects of different parameters such as the mass of the load, the length of the moving load, the distance covered by the moving load, the speed of the moving and the axial force were considered. Result revealed that the values of the deflection with acceleration being considered are higher than the system where acceleration of the moving load is negligible. These obtained results are in agreement with the existing results.
\end{abstract}

Keywords: elastic beam, axial force, accelerated distributed load, dynamic response

\section{INTRODUCTION}

Most physical and engineering Boundary Value Problem (BVP) can be modelled as functional equations. However, for most of these equations, exact solutions are very rare. Several analytical and numerical methods are being developed to obtain approximate solutions for such models. ${ }^{1,2}$ Euler-Bernoulli beam is one of the models which is a distributed load. Analytical solutions for BVPs are always preferable compared to numerical solutions as they are more general and give a better understanding of the model behaviour. On the other hand, unfortunately, analytical solutions are limited to simple and idealised models. Due to great practical importance for technical engineers, the analysis of the 
dynamic behaviour of beams on elastic foundations has attracted many researchers in the century and has therefore been the object of study by a huge number of researchers until now. ${ }^{3-12}$

The exact theoretical solutions of undamped vibrations for a simply supported Euler-Bernoulli beam was examined. ${ }^{10}$ The problem of the dynamic response of beams connected viscoelastically and subjected to uniform partially distributed moving force was studied. ${ }^{11}$ Result indicated that there was an increase in the amplitude of the beam for various values of the speed of the moving force considered, such that, as the speed of the moving force increases, the amplitude of the beam also increases. Furthermore, Oni and Ayankop-Andi ${ }^{8}$ considered the dynamic response of a Bernoulli-beam with viscous and structural damping coefficients subjected to partially distributed moving load. It was observed that as the mass of the beam, increases, the deflection also increases. However, the amplitude of the deflection increases when there are no structural and damping coefficients.

From the following literature, studies have only considered the moving load problem at constant speed, which can be traced to simplicity in solving compared to when acceleration is considered. However, the study of the effect of acceleration on the moving load problem is very scanty. But, it is necessary to put into consideration, the acceleration of the moving load. The analytical solution was employed to solve the partially distributed moving load taking acceleration into consideration. We assumed a series solution in the governing equation in order to transform the fourth order partial differential equation into order two.

Hence, in this article, the dynamic response of Euler-Bernoulli beam subjected to accelerated moving load was investigated.

\section{MATERIALS AND METHODS}

In this article, a BVP under subjected to distributed moving load resting on a Winkler foundation is considered. As shown in Figure 1, the beam equation which is an example of a BVP of length $(l)$, a uniform cross-sectional area $(A)$, a cross-sectional moment of inertia $(I)$, modulus of elasticity $(E)$ and density $(\rho)$. 


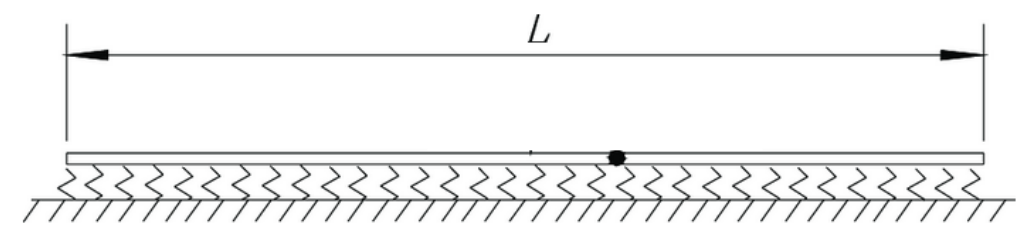

Figure 1: Beam structure.

The model for the problem under consideration is relatively simple Winkler model. According to theory of structural vibration on using Euler-Bernoulli beam. ${ }^{2}$ The governing equation is formulated as:

$$
E I \frac{\partial^{4} \psi(x, t)}{\partial x^{4}}+m \frac{\partial^{2} \psi(x, t)}{\partial x^{2}}-\rho_{0} \frac{\partial^{2} \psi(x, t)}{\partial x^{2}}+K \psi(x, t)=f(x, t)
$$

where

$$
f(x, t)=\frac{1}{\varepsilon}\left[-M g-M \frac{\partial^{2} \psi}{\partial x^{2}}\right] B
$$

and

$$
B=\delta(x-v t)=\frac{d H(x)}{d t^{2}}=\left(H\left(x-\xi+\frac{\varepsilon}{2}\right)-H\left(x-\xi-\frac{\varepsilon}{2}\right)\right)
$$

In Equation 1, the one parameter $(K)$ was used to model the elastic support based on classical Winkler model.

\subsection{Classical Boundary Conditions}

The initial condition of the beam at rest is given as:

$$
\left.\begin{array}{r}
\psi(x, 0)=0 \\
\frac{d \psi(x, 0)}{d t}=0
\end{array}\right\}
$$


and the pertinent boundary conditions for the problem under consideration are:

$$
\left.\begin{array}{r}
\psi(x, t)=\frac{\partial \psi(x, t)}{\partial x}=0 \text { at } x=0 \text { or } x=L \\
\psi(x, t)=\left.\frac{\partial^{2} \psi(x, t)}{\partial x^{2}}\right|_{x=0}=0 \text { at } x=0 \text { or } x=L \\
\frac{\partial^{2} \psi(x, t)}{\partial x^{2}}=\frac{\partial^{3} \psi(x, t)}{\partial x^{3}}=0 \text { at } x=0 \text { or } x=L \\
\frac{\partial \psi(x, t)}{\partial x}=\frac{\partial^{3} \psi(x, t)}{\partial x^{3}}=0 \text { at } x=0 \text { or } x=L
\end{array}\right\}
$$

The external load in Equation 1 is defined by:

$$
f(x, t)=\frac{1}{\varepsilon}\left[-M g-M \frac{\partial^{2} \psi}{\partial x^{2}}\right] B
$$

where

$$
B=\delta(x-v t)=\frac{d H(x)}{d t^{2}}
$$

The reaction force $(f)$ exerted by mass $(m)$ is defined as:

$$
\begin{aligned}
\frac{\partial^{2} \psi\left(x_{f}, t\right)}{\partial t^{2}} & =\frac{\partial^{2} \psi(x, t)}{\partial t^{2}}+2 V \frac{\partial^{2} \psi(x, t)}{\partial t \partial x}+V^{2} \frac{\partial^{2} \psi(x, t)}{\partial x^{2}} \\
& =\frac{\partial^{2} \psi\left(x_{f}, t\right)}{\partial t^{2}}+2\left(\frac{\partial x_{f}(x, t)}{\partial t}\right) \frac{\partial^{2} \psi(x, t)}{\partial t \partial x}+\left(\frac{\partial x_{f}}{\partial t}\right)^{2} \frac{\partial^{2} \psi(x, t)}{\partial x^{2}}
\end{aligned}
$$

where

$$
\begin{aligned}
& x_{f}(t)=x_{0}+\ddot{x}_{0} t+\frac{a_{n} t^{2}}{2} \\
& \frac{d x_{f}(t)}{d t}=\ddot{x}+a_{n} t \\
& \frac{d^{2} x_{f}(t)}{d t^{2}}=a_{n}
\end{aligned}
$$


Substituting Equations 8, 9 and 10 into Equation 1 to obtain:

$$
\begin{gathered}
E I \frac{\partial^{4} \psi(x, t)}{\partial x^{4}}+m \frac{\partial^{2} \psi(x, t)}{\partial t^{2}}-\rho_{0} \frac{\partial^{2} \psi(x, t)}{\partial x^{2}}+K \psi(x, t) \\
=\left(\frac{1}{\varepsilon}\right)\left(-M g-M \frac{\partial^{2} \psi}{\partial x^{2}}\right) B \\
E I \frac{\partial^{4} \psi(x, t)}{\partial x^{4}}+m \frac{\partial^{2} \psi(x, t)}{\partial t^{2}}-\rho_{0} \frac{\partial^{2} \psi(x, t)}{\partial x^{2}}+K \psi(x, t) \\
=\left(\frac{1}{\varepsilon}\right)\left[-M\left(g-\left(\frac{\partial^{2} \psi(x, t)}{\partial t^{2}}+2\left(\ddot{x}+a_{n} t\right) \frac{\partial^{2} \psi(x, t)}{\partial t \partial x}+a_{n} \frac{\partial^{2} \psi(x, t)}{\partial x^{2}}\right)\right] B\right.
\end{gathered}
$$

\subsection{Solution Techniques}

In this section, we proceed to solve the above initial BVP described by Equation 12. Assume a solution of the form as:

$$
\psi(x, t)=\sum_{j=1}^{\infty} q_{j}(t) \sin \frac{j \pi x}{L}
$$

Differentiating Equation 14 with respect to $x$ and with respect to $t$,

$$
\frac{\partial^{4} \psi(x, t)}{\partial x^{4}}=\left(\frac{j \pi}{L}\right)^{4} \sum_{j=1}^{\infty} j^{4} q_{j}(t) \sin \frac{j \pi x}{L}
$$

Also,

$$
\frac{\partial^{2} \psi(x, t)}{\partial t^{2}}=\sum_{j=1}^{\infty} \ddot{q}_{j}(t) \sin \frac{j \pi x}{L}
$$

Next,

$$
\frac{\partial^{2} \psi(x, t)}{\partial t^{2}}=\left(\frac{j \pi}{L}\right)^{2} \sum_{j=1}^{\infty} j^{2} q_{j}(t) \sin \frac{j \pi x}{L}
$$


Substituting Equations 14, 15, 16 and 17 into Equation 13, we have:

$$
\begin{gathered}
E I\left(\frac{j \pi}{L}\right)^{4} \sum_{j=1}^{\infty} j^{4} q_{j}(t) \sin \frac{j \pi x}{L}+m \sum_{j=1}^{\infty} \ddot{q}_{j}(t) \sin \frac{j \pi x}{L} \\
-\rho_{0}\left(\frac{j \pi}{L}\right)^{2} \sum_{j=1}^{\infty} j^{2} q_{j}(t) \sin \frac{j \pi x}{L}+K \sum_{j=1}^{\infty} q_{j}(t) \sin \frac{j \pi x}{L} \\
=\left(\frac{1}{\epsilon}\right)\left[-M\left(g-\left(\sum_{j=1}^{\infty} \ddot{q}_{j}(t) \sin \frac{j \pi x}{L}\right.\right.\right. \\
+2\left(\ddot{x}+a_{n} t\right)\left(\frac{j \pi}{L}\right) \sum_{j=1}^{\infty} \dot{q}_{j}(t) \cos \frac{j \pi x}{L} \\
\left.\left.+a_{n}\left(\frac{j \pi}{L}\right)^{2} \sum_{j=1}^{\infty} j^{2} q_{j}(t) \sin \frac{j \pi x}{L}\right)\right] B
\end{gathered}
$$

But,

$$
f(x, t)=\left(\frac{1}{\epsilon}\right)\left[-M B-\left(\begin{array}{l}
\sum_{j=1}^{\infty} \ddot{q}_{j}(t) \sin \frac{j \pi x}{L} \\
+2\left(\ddot{x}+a_{n} t\right)\left(\frac{j \pi}{L}\right) \sum_{j=1}^{\infty} \dot{q}_{j}(t) \cos \frac{j \pi x}{L} \\
+a_{n}\left(\frac{j \pi}{L}\right)^{2} \sum_{j=1}^{\infty} j^{2} q_{j}(t) \sin \frac{j \pi x}{L}
\end{array}\right) B\right.
$$

Equation 19 becomes:

$$
\begin{aligned}
f(x, t)= & \left(\frac{1}{\epsilon}\right)\left[-M\left(g(B)-\left(\sum_{j=1}^{\infty} \ddot{q}_{j}(t) \sin \frac{j \pi x}{L}\right.\right.\right. \\
& +2\left(\ddot{x}+a_{n} t\right)\left(\frac{j \pi}{L}\right) \sum_{j=1}^{\infty} \dot{q}_{j}(t) \cos \frac{j \pi x}{L} \\
& \left.\left.+a_{n}\left(\frac{j \pi}{L}\right)^{2} \sum_{j=1}^{\infty} j^{2} q_{j}(t) \sin \frac{j \pi x}{L}\right) B\right]
\end{aligned}
$$

Let

$$
f(x, t)=\sum_{j=1}^{\infty} q_{f j}(t) \sin \frac{j \pi x}{L}
$$


Also, the applied force can be:

$$
\sum_{j=1}^{\infty} q_{f j}(t) \sin \frac{j \pi x}{L}=\left(\frac{1}{\epsilon}\right)\left[(-M g B)-\left(\begin{array}{l}
\sum_{j=1}^{\infty} \ddot{q}_{j}(t) \sin \frac{j \pi x}{L} \\
+2\left(\ddot{x}+a_{n} t\right)\left(\frac{j \pi}{L}\right) \sum_{j=1}^{\infty} \dot{q}_{j}(t) \cos \frac{j \pi x}{L} \\
+a_{n}\left(\frac{j \pi}{L}\right)^{2} \sum_{j=1}^{\infty} j^{2} q_{j}(t) \sin \frac{j \pi x}{L}
\end{array}\right] B\right]
$$

Multiply Equation 22 by $\frac{i \pi x}{L}$ and integrate, we have:

$$
\begin{aligned}
\sum_{j=1}^{\infty} q_{f j}(t) \int_{0}^{L} \sin \frac{i \pi x}{L}= & -\frac{M}{\epsilon}\left(\int_{0}^{L} \sin \frac{i \pi x}{L} B\right) d x \\
& -\frac{M}{\epsilon}\left(\int_{0}^{L} \sin \frac{i \pi x}{L} B\right) d x\left(\sum_{j=1}^{\infty} \ddot{q}_{j}(t) \int_{0}^{L} \sin \frac{i \pi x}{L} \sin \frac{j \pi x}{L} B\right) d x \\
& -\frac{2 M}{\epsilon} L_{11} \sum_{j=1}^{\infty} \dot{q}_{j}(t) \int_{0}^{L} \sin \frac{i \pi x}{L} \cos \frac{j \pi x}{L} B d x \\
& -\frac{M}{\epsilon} L_{12} \sum_{j=1}^{\infty} j^{2} q_{j}(t) \int_{0}^{L} \sin \frac{i \pi x}{L} \sin \frac{j \pi x}{L} B d x
\end{aligned}
$$

where $L_{11}$ represent $\left(\ddot{x}+a_{n} t\right)\left(\frac{j \pi}{L}\right)$

$$
L_{22} \text { represent } a_{n}\left(\frac{\pi}{L}\right)^{2}
$$

e.g.,

$$
Z_{15}=Z_{11}+Z_{12}+Z_{13}+Z_{14}
$$

where

$$
\begin{aligned}
& Z_{11}=-\frac{M g}{\epsilon} \int_{0}^{L} \sin \frac{i \pi x}{L} B d x \\
& Z_{12}=-\frac{M}{\epsilon}\left(\sum_{j=1}^{\infty} \ddot{q}_{j}(t) \int_{0}^{L} \sin \frac{i \pi x}{L} \sin \frac{j \pi x}{L} B\right) d x
\end{aligned}
$$




$$
\begin{aligned}
& Z_{13}=-\frac{2 M}{\epsilon} L_{11} \sum_{j=1}^{\infty} \dot{q}_{j}(t) \int_{0}^{L} \sin \frac{i \pi x}{L} \cos \frac{j \pi x}{L} B d x \\
& Z_{14}=-\frac{M}{\epsilon} L_{12} \sum_{j=1}^{\infty} j^{2} q_{j}(t) \int_{0}^{L} \sin \frac{i \pi x}{L} \sin \frac{j \pi x}{L} B d x
\end{aligned}
$$

and

$$
Z_{15}=\sum_{j=1}^{\infty} q_{f j}(t) \int_{0}^{L} \sin \frac{i \pi x}{L} d x
$$

In evaluating the integrals in Equations 25(b) to 25(f), we made use of the following:

$$
\begin{aligned}
& H\left(x-\left(\xi-\frac{\epsilon}{2}\right)\right)= \begin{cases}0, & \text { if } x<\xi-\frac{\epsilon}{2} \\
1, & \text { if } x>\xi-\frac{\epsilon}{2}\end{cases} \\
& H\left(x-\left(\xi+\frac{\epsilon}{2}\right)\right)= \begin{cases}0, & \text { if } x<\xi+\frac{\epsilon}{2} \\
1, & \text { if } x>\xi-\frac{\epsilon}{2}\end{cases}
\end{aligned}
$$

By making use of the Dirac delta function, Equations 25(b) to 25(f) become:

$$
\begin{aligned}
Z_{11}= & -\frac{M g}{\epsilon} \int_{0}^{L} \sin \frac{i \pi}{L}\left(\xi+\frac{\epsilon}{2}\right) d \epsilon-\sin \frac{i \pi}{L}\left(\xi-\frac{\epsilon}{2}\right) d \epsilon \\
Z_{12}= & -\frac{M}{\epsilon} \sum_{j=1}^{\infty} \ddot{q}_{j}(t) \int_{0}^{L}\left[\sin \frac{i \pi}{L}\left(\xi+\frac{\epsilon}{2}\right) \sin \frac{i \pi}{L}\left(\xi-\frac{\epsilon}{2}\right)\right. \\
& \left.-\sin \frac{j \pi}{L}\left(\xi+\frac{\epsilon}{2}\right) \sin \frac{j \pi}{L}\left(\xi-\frac{\epsilon}{2}\right)\right] d \epsilon \\
Z_{13}= & -\frac{2 M}{\epsilon} L_{11} \sum_{j=1}^{\infty} \dot{q}_{j}(t) \int_{0}^{L}\left[\sin \frac{i \pi}{L}\left(\xi+\frac{\epsilon}{2}\right)\right. \\
& \left.-\sin \frac{i \pi}{L}\left(\xi-\frac{\epsilon}{2}\right) \cos \frac{j \pi}{L}\left(\xi+\frac{\epsilon}{2}\right)-\cos \frac{j \pi}{L}\left(\xi-\frac{\epsilon}{2}\right)\right] d \epsilon
\end{aligned}
$$




$$
\begin{aligned}
Z_{14}= & -\frac{M}{\epsilon} L_{12} \sum_{j=1}^{\infty} j^{2} q_{j}(t) \int_{0}^{L}\left[\sin \frac{i \pi}{L}\left(\xi+\frac{\epsilon}{2}\right)\right. \\
& \left.-\sin \frac{i \pi}{L}\left(\xi-\frac{\epsilon}{2}\right) \sin \frac{j \pi}{L}\left(\xi+\frac{\epsilon}{2}\right) \sin \frac{j \pi}{L}\left(\xi-\frac{\epsilon}{2}\right)\right] d \epsilon
\end{aligned}
$$

and

$$
Z_{15}=q_{f j}(t)
$$

Evaluating the integrals in Equations 26(a) to 26(e), we finally obtained:

$$
\begin{aligned}
q_{f j}(t)= & -\frac{2 M g L}{j \pi \epsilon} \sin \frac{i \pi \xi}{L} \sin \frac{i \pi \epsilon}{2 L} \\
& -\frac{2 M}{j \pi \epsilon} \sum_{j=1}^{\infty} \ddot{q}_{j}(t) \frac{1}{b_{12}}\left[\sin \frac{i \pi \xi}{2 L} b_{12} \cos \frac{\pi \epsilon}{L} b_{12}\right]-\frac{1}{b_{12}}\left(\cos \frac{\pi \xi}{L} b_{11} \sin \frac{\pi \epsilon}{2 L} b_{11}\right) \\
& -\frac{2 M}{\epsilon} L_{11} \sum_{j=1}^{\infty} \dot{q}_{j}(t)\left[\sin \frac{\pi \xi}{L} b_{11} \sin \frac{\pi \epsilon}{2 L} b_{11}+\frac{1}{b_{12}} \sin \frac{\pi \xi}{L} b_{12} \sin \frac{\pi \epsilon}{2 L} b_{12}\right] \\
& -\frac{M}{\epsilon} L_{12} \sum_{j=1}^{\infty} q_{j}(t) \frac{1}{b_{11}}\left[\sin \frac{\pi \xi}{L} b_{11} \cos \frac{\pi \epsilon}{2 L} b_{11}+\cos \frac{\pi \xi}{L} b_{11} \sin \frac{\pi \epsilon}{2 L} b_{11}\right]
\end{aligned}
$$

Substituting Equations 26(a) to 26(d) and 27 into Equation 23:

$$
\begin{aligned}
E I L_{13} \sum_{j=1}^{\infty} q_{j}(t) \sin \frac{j \pi x}{L}+m \sum_{j=1}^{\infty} \ddot{q}_{j}(t) \sin \frac{j \pi x}{L}-\rho_{0} L_{14} \sum_{j=1}^{\infty} q_{j}(t) \sin \frac{j \pi x}{L} \\
+K \sum_{j=1}^{\infty} q_{j}(t) \sin \frac{j \pi x}{L} \\
=\sum_{j=1}^{\infty} \sin \frac{j \pi x}{L}\left[-\frac{2 M g L}{j \pi \epsilon} \sin \frac{i \pi \xi}{L} \sin \frac{i \pi \epsilon}{2 L}\right. \\
-\frac{2 M}{j \pi \epsilon} \sum_{j=1}^{\infty} \ddot{q}_{j}(t) \frac{1}{b_{12}}\left[\sin \frac{i \pi \xi}{L} b_{12} \cos \frac{\pi \epsilon}{L} b_{12}\right]-\frac{1}{b_{12}}\left(\cos \frac{\pi \xi}{L} b_{11} \sin \frac{\pi \epsilon}{2 L} b_{11}\right) \\
\quad-\frac{2 M}{\epsilon} L_{11} \sum_{j=1}^{\infty} \dot{q}_{j}(t)\left[\sin \frac{\pi \xi}{L} b_{11} \sin \frac{\pi \epsilon}{2 L} b_{11}+\frac{1}{b_{12}} \sin \frac{\pi \xi}{L} b_{12} \sin \frac{\pi \epsilon}{2 L} b_{12}\right] \\
\left.-\frac{M}{\epsilon} L_{12} \sum_{j=1}^{\infty} q_{j}(t) \frac{1}{b_{11}}\left[\sin \frac{\pi \xi}{L} b_{11} \cos \frac{\pi \epsilon}{2 L} b_{11}+\cos \frac{\pi \xi}{L} b_{11} \sin \frac{\pi \epsilon}{2 L} b_{11}\right]\right]
\end{aligned}
$$


where $L_{13}$ represents $\left(\frac{\pi}{L}\right)^{4}, L_{14}$ represents $\left(\frac{\pi}{L}\right)^{2}, b_{11}$ represents $i+j$ and $b_{12}$ represents $i-j$. Simplifying Equation 28, we have:

$$
\begin{aligned}
& \sum_{j=1}^{\infty}\left[\left[E I L_{13} q_{j}(t)+m \ddot{q}_{j}(t)-\rho_{0} L_{14} q_{j}(t)+K q_{j}(t)-\left[-\frac{2 M g L}{j \pi \epsilon} \sin \frac{i \pi \xi}{L} \sin \frac{i \pi \epsilon}{2 L}\right.\right.\right. \\
& -\frac{2 M}{\epsilon \pi} \sum_{j=1}^{\infty} \ddot{q}_{j}(t) \frac{1}{b_{12}}\left[\sin \frac{i \pi \xi}{L} b_{12} \cos \frac{\pi \epsilon}{L} b_{12}\right]-\frac{1}{b_{12}}\left(\cos \frac{\pi \xi}{L} b_{11} \sin \frac{\pi \epsilon}{2 L} b_{11}\right) \\
& -\frac{2 M}{\epsilon} L_{11} \sum_{j=1}^{\infty} \dot{q}_{j}(t)\left[\sin \frac{\pi \xi}{L} b_{11} \sin \frac{\pi \epsilon}{2 L} b_{11}+\frac{1}{b_{12}} \sin \frac{\pi \xi}{L} b_{12} \sin \frac{\pi \epsilon}{2 L} b_{12}\right] \\
& \left.\left.-\frac{M}{\epsilon} L_{12} \sum_{j=1}^{\infty} q_{j}(t) \frac{1}{b_{11}}\left[\sin \frac{\pi \xi}{L} b_{11} \cos \frac{\pi \epsilon}{2 L} b_{11}+\cos \frac{\pi \xi}{L} b_{11} \sin \frac{\pi \epsilon}{2 L} b_{11}\right]\right]\right] \\
& \sin \frac{j \pi x}{L}=0, \quad j=1,2,3, \quad i \neq j
\end{aligned}
$$

which implies that

$$
\begin{aligned}
m \ddot{q}_{j}(t) & +\left(E I L_{13}+m-\rho_{0} L_{14}\right) q_{j}(t) \\
& =-\frac{2 M g L}{j \pi \epsilon} \sin \frac{i \pi \xi}{L} \sin \frac{i \pi \epsilon}{2 L}-\frac{2 M}{\epsilon \pi} \sum_{j=1}^{\infty} \ddot{q}_{j}(t) \frac{1}{b_{12}}\left[\sin \frac{i \pi \xi}{2 L} b_{12} \cos \frac{\pi \epsilon}{L} b_{12}\right] \\
& -\frac{1}{b_{12}}\left(\cos \frac{\pi \xi}{L} b_{11} \sin \frac{\pi \epsilon}{2 L} b_{11}\right) \\
& -\frac{2 M}{\epsilon} L_{11} \sum_{j=1}^{\infty} \dot{q}_{j}(t)\left[\sin \frac{\pi \xi}{L} b_{11} \sin \frac{\pi \epsilon}{2 L} b_{11}+\frac{1}{b_{12}} \sin \frac{\pi \xi}{L} b_{12} \sin \frac{\pi \epsilon}{2 L} b_{12}\right] \\
& -\frac{M}{\epsilon} L_{12} \sum_{j=1}^{\infty} q_{j}(t) \frac{1}{b_{11}}\left[\sin \frac{\pi \xi}{L} b_{11} \cos \frac{\pi \epsilon}{2 L} b_{11}+\cos \frac{\pi \xi}{L} b_{11} \sin \frac{\pi \epsilon}{2 L} b_{11}\right]
\end{aligned}
$$


Rearranging Equation 30, we have:

$$
\begin{aligned}
m \ddot{q}_{j}(t) & +L_{15} q_{j}(t) \\
& =-\frac{2 M g L}{j \pi \epsilon} \sin \frac{i \pi \xi}{L} \sin \frac{i \pi \epsilon}{2 L} \\
& -\frac{2 M}{\epsilon \pi} \sum_{j=1}^{\infty} \ddot{q}_{j}(t) \frac{1}{b_{12}}\left[\sin \frac{i \pi \xi}{2 L} b_{12} \cos \frac{\pi \epsilon}{L} b_{12}\right]-\frac{1}{b_{12}}\left(\cos \frac{\pi \xi}{L} b_{11} \sin \frac{\pi \epsilon}{2 L} b_{11}\right) \\
& -\frac{2 M}{\epsilon} L_{11} \sum_{j=1}^{\infty} \dot{q}_{j}(t)\left[\sin \frac{\pi \xi}{L} b_{11} \sin \frac{\pi \epsilon}{2 L} b_{11}+\frac{1}{b_{12}} \sin \frac{\pi \xi}{L} b_{12} \sin \frac{\pi \epsilon}{2 L} b_{12}\right] \\
& -\frac{M}{\epsilon} L_{12} \sum_{j=1}^{\infty} q_{j}(t) \frac{1}{b_{11}}\left[\sin \frac{\pi \xi}{L} b_{11} \cos \frac{\pi \epsilon}{2 L} b_{11}+\cos \frac{\pi \xi}{L} b_{11} \sin \frac{\pi \epsilon}{2 L} b_{11}\right]
\end{aligned}
$$

where $L_{15}=E I L_{13}+m-\rho_{0} L_{14}$.

\section{NUMERICAL RESULTS AND DISCUSSION}

Numerical computation has been carried out by using the central difference techniques. Applying this numerical method to derivatives in Equation 31, we obtained:

$$
\begin{aligned}
& m\left(\frac{q_{j+1}-2 q_{j}+q_{j-1}}{h^{2}}\right)+L_{15} q_{j} \\
& =-\frac{2 M g L}{j \pi \epsilon} \sin \frac{i \pi \xi}{L} \sin \frac{i \pi \epsilon}{2 L}-\frac{2 M}{\epsilon \pi}\left(\frac{q_{b_{11}}-2 q_{j}+q b_{12}}{h^{2}}\right) \\
& \frac{1}{b_{12}}\left[\sin \frac{i \pi \xi}{2 L} b_{12} \cos \frac{\pi \epsilon}{L} b_{12}-\frac{1}{b_{12}}\left(\cos \frac{\pi \xi}{L} b_{11} \sin \frac{\pi \epsilon}{2 L} b_{11}\right)\right] \\
& -\frac{2 M}{\epsilon} L_{11}\left(\frac{q_{b_{11}}-q_{b_{12}}}{2 h}\right)\left[\sin \frac{\pi \xi}{L} b_{11} \sin \frac{\pi \epsilon}{2 L} b_{11}+\frac{1}{b_{12}} \sin \frac{\pi \xi}{L} b_{12} \sin \frac{\pi \epsilon}{2 L} b_{12}\right] \\
& -\frac{M}{\epsilon} L_{12} q_{j} \frac{1}{b_{11}}\left[\sin \frac{\pi \xi}{L} b_{11} \cos \frac{\pi \epsilon}{2 L} b_{11}+\cos \frac{\pi \xi}{L} b_{11} \sin \frac{\pi \epsilon}{2 L} b_{11}\right]
\end{aligned}
$$

$j=1,2,3, \quad i \neq j$ 
Simplifying Equation 32, we finally obtain:

$$
\begin{aligned}
-\frac{2 M g L h^{2}}{j \pi \epsilon} \sin \left(\frac{i \pi \xi}{L}\right) \sin \left(\frac{i \pi \epsilon}{2 L}\right) \\
=\left[m+\frac{2 M}{\epsilon \pi} \frac{1}{b_{12}}\left[\sin \frac{i \pi \xi}{2 L} b_{12} \cos \frac{\pi \epsilon}{L} b_{12}-\frac{1}{b_{12}}\left(\cos \frac{\pi \xi}{L} b_{11} \sin \frac{\pi \epsilon}{2 L} b_{11}\right)\right]\right. \\
\left.-\quad \frac{2 M}{\epsilon} L_{11} h\left[\sin \frac{\pi \xi}{L} b_{11} \sin \frac{\pi \epsilon}{2 L} b_{11}+\frac{1}{b_{12}} \sin \frac{\pi \xi}{L} b_{12} \sin \frac{\pi \epsilon}{2 L} b_{12}\right]\right] q_{b_{11}} \\
+\left[\left(-2\left(m+h^{2} L_{15}\right)-\frac{4 m j}{\epsilon \pi} \frac{1}{b_{12}}\left(\sin \frac{\pi \xi}{L} b_{12} \cos \frac{\pi \epsilon}{2 L} b_{12}\right)\right.\right. \\
\left.\quad-\frac{1}{b_{11}} \cos \frac{\pi \xi}{L} b_{11} \sin \frac{\pi \epsilon}{2 L} b_{11}\right)-\frac{m}{\epsilon} L_{12} \frac{1}{b_{11}}\left(\sin \frac{\pi \xi}{L} b_{11} \cos \frac{\pi \epsilon}{2 L} b_{11}\right. \\
\left.\left.+\cos \frac{\pi \xi}{L} b_{11} \sin \frac{\pi \epsilon}{2 L} b_{11}\right)\right] q_{j}+\left[m+\frac{2 M}{\epsilon \pi} \frac{1}{b_{12}}\left(\sin \frac{\pi \xi}{L} b_{12} \cos \frac{\pi \epsilon}{2 L} b_{12}\right)\right. \\
+\frac{1}{b_{11}}\left(\cos \frac{\pi \xi}{L} b_{11} \sin \frac{\pi \epsilon}{2 L} b_{11}\right)+\frac{1}{b_{11}} \frac{m L_{11} h j}{\epsilon \pi}\left(\sin \frac{\pi \xi}{L} b_{11} \sin \frac{\pi \epsilon}{2 L} b_{11}\right. \\
\left.\left.+\frac{1}{b_{11}} \sin \frac{\pi \xi}{L} b_{12} \sin \frac{\pi \epsilon}{2 L} b_{12}\right)\right] q_{b_{12}} \mathrm{q} \\
j=1,2,3, \quad i \neq j
\end{aligned}
$$

\section{RESULTS AND DISCUSSION}

In this section, the following parameter were used for the numerical analysis: $E I=1.74 \times 10^{-5}, L=10 \mathrm{~m}, j=2, i=2, \rho_{0}=5, \xi=0.12, v=3.3 \mathrm{~m} \mathrm{~s}^{-2}, M=50 \mathrm{~kg}$, $m=984 \mathrm{~kg} \mathrm{~m}^{-2}$. The final results obtained for the problem under consideration is programmed in the MATLAB environment.

Figures 2(a) and 2(b) show the deflection of beam for different values of the mass of the load $(M)$ for which the acceleration of the moving load is considered and neglected, respectively. It is observed that the deflection is increased by increasing the values of the $M$. The values of the deflection with acceleration being considered are higher than the system where acceleration of the moving load is negligible. 


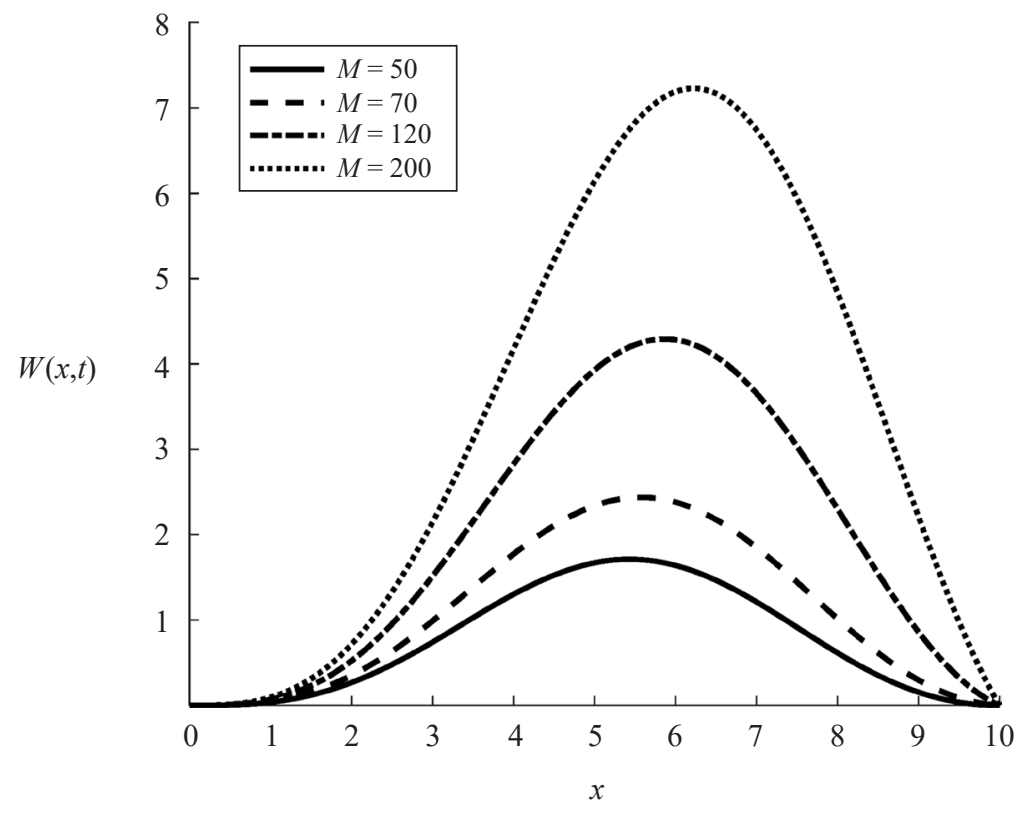

Figure 2(a): Deflection of beam at various values of $M$ (acceleration considered).

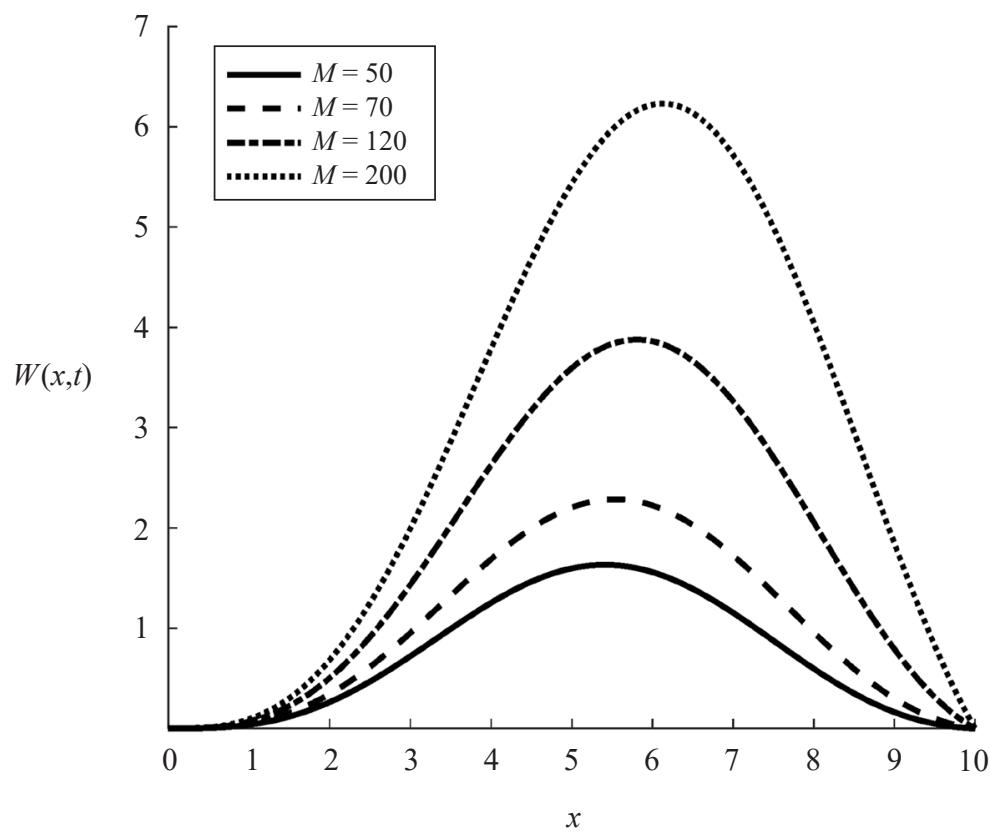

Figure 2(b): Deflection for beam at various values of $M$ (acceleration neglected). 
On the other hand, Figures 3(a) and 3(b) show the deflection of beam for different values of the speed of the moving load $(v)$ for which the acceleration of the moving load is considered and neglected, respectively. It is observed that the deflection increases as the values of $v$ is increasing. It is seen that the values of the deflection with acceleration being considered is higher than the system where acceleration of the moving load is negligible.

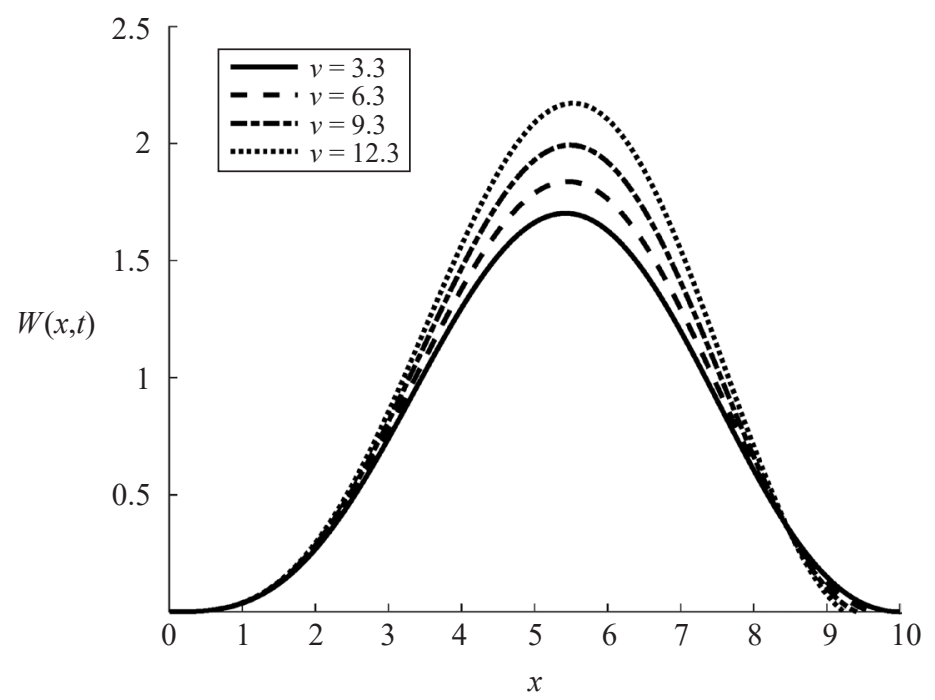

Figure 3(a): Deflection of beam at various values of $v$ (acceleration considered).

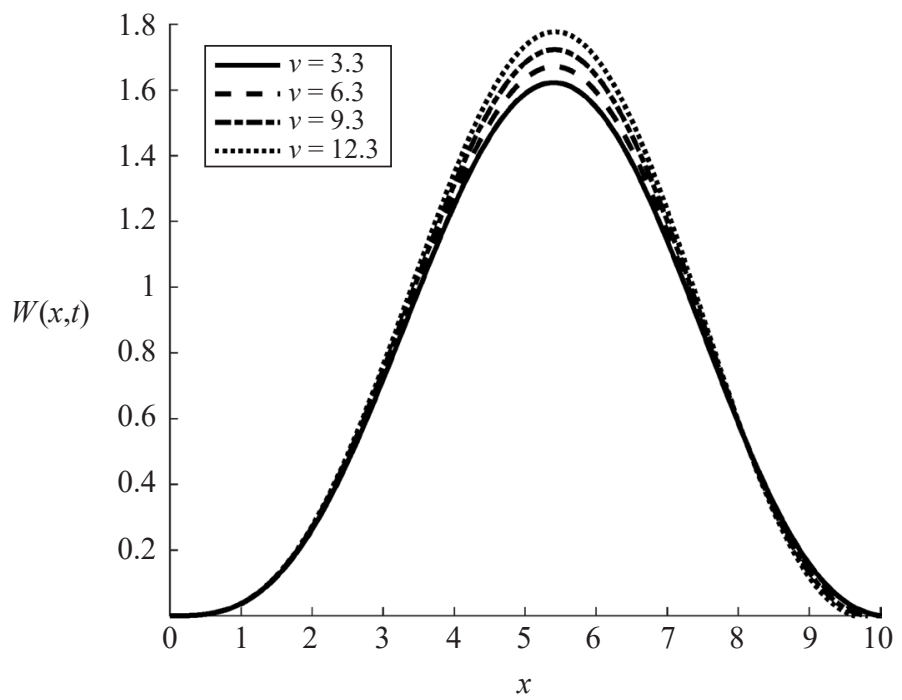

Figure 3(b): Deflection of beam for various values of $v$ (acceleration neglected). 
The deflection of beam for different values of the length of the load $(\xi)$ for which the acceleration of the moving load is considered and neglected are shown in Figures 4(a) and 4(b), respectively. It is observed that the deflection is increased by increasing the values of $\xi$ and the values of the deflection with acceleration being considered is higher than the system where acceleration of the moving load is negligible.

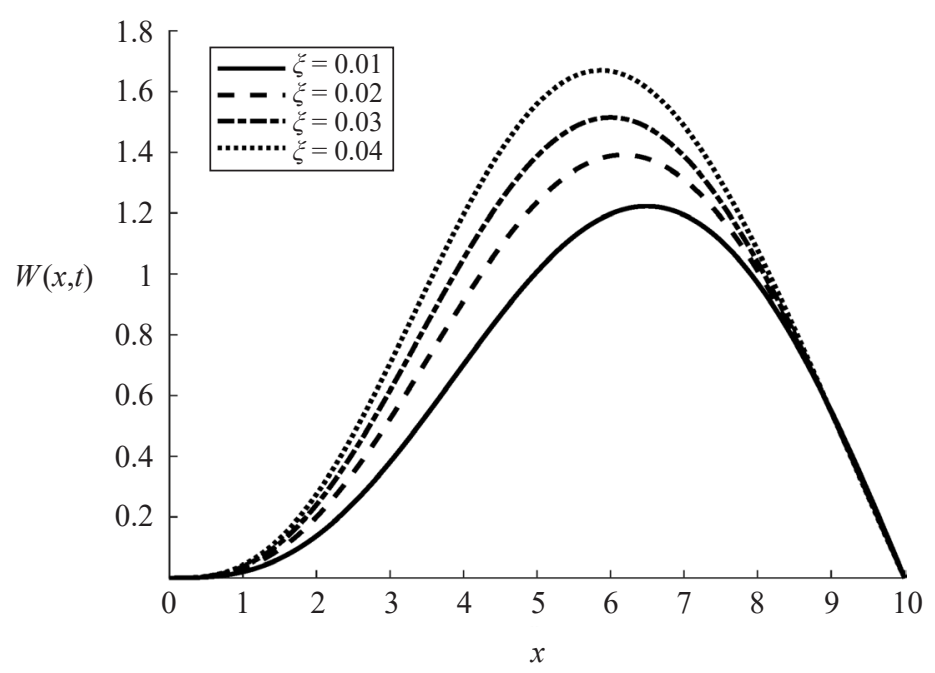

Figure 4(a): Deflection of beam for various values of $\xi$ (acceleration considered).

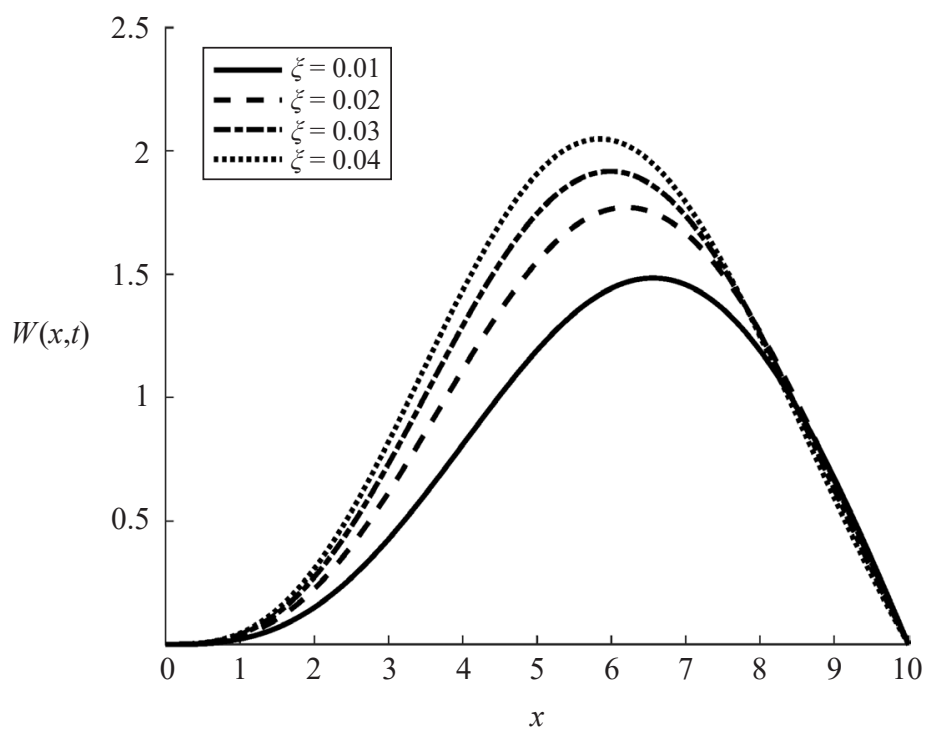

Figure 4(b): Deflection of beam for various values of $\xi$ (acceleration neglected). 
For Figures 5(a) and 5(b), they show the deflection of beam for different values of the distance moved of the load $(\epsilon)$ for which the acceleration of the moving load is considered and neglected, respectively. It is observed that the deflection increases as the values of $\epsilon$ is increasing. It is seen that the values of the deflection with acceleration being considered is higher than the system where acceleration of the moving load is negligible.

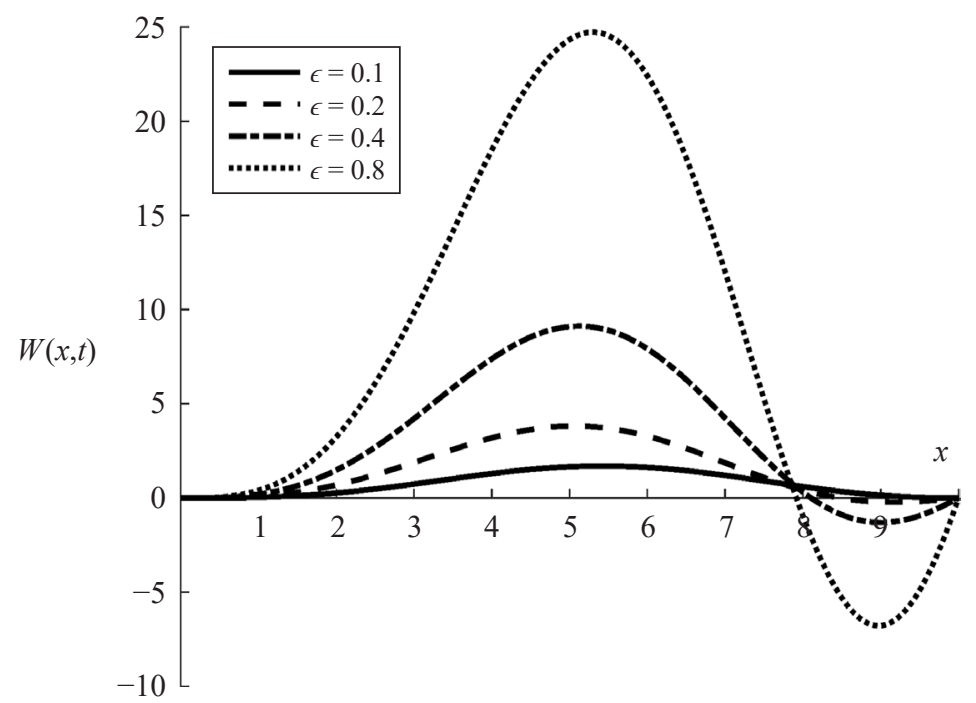

Figure 5(a): Deflection of beam for various values of $\epsilon$ (acceleration considered).

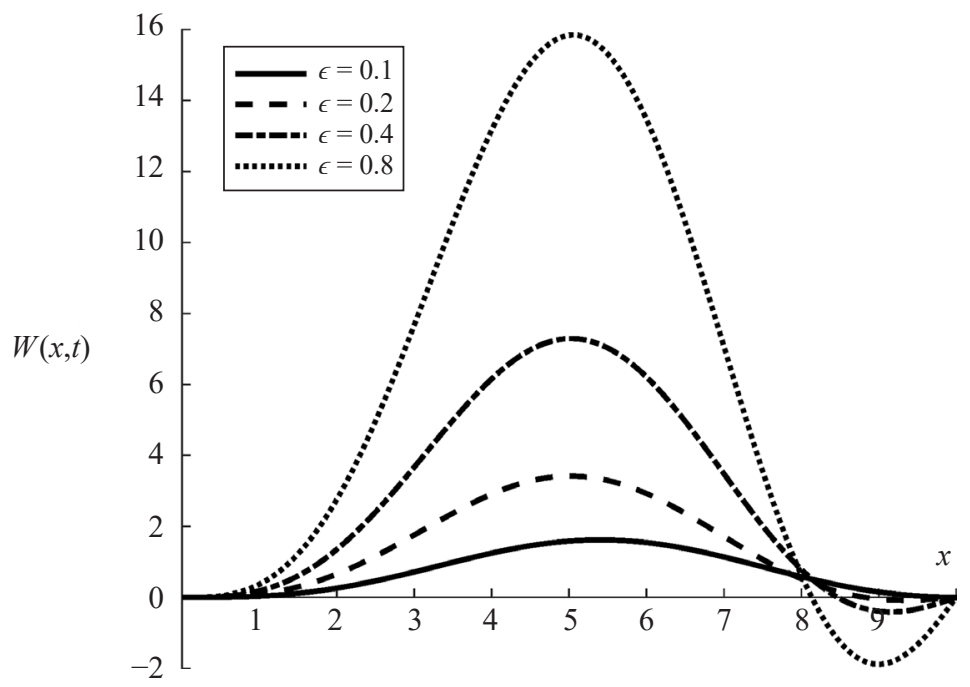

Figure 5(b): Deflection of beams for various values of $\epsilon$ (acceleration neglected). 
Apart from that, the deflection of beam for different values of the axial force $\left(\rho_{0}\right)$ which the acceleration of the moving load is considered and neglected are shown in Figures 6(a) and 6(b), respectively. It is observed that the deflection is increased by increasing the values of $\rho_{0}$. It is seen that the values of the deflection with acceleration being considered is higher than the system where acceleration of the moving load is negligible.

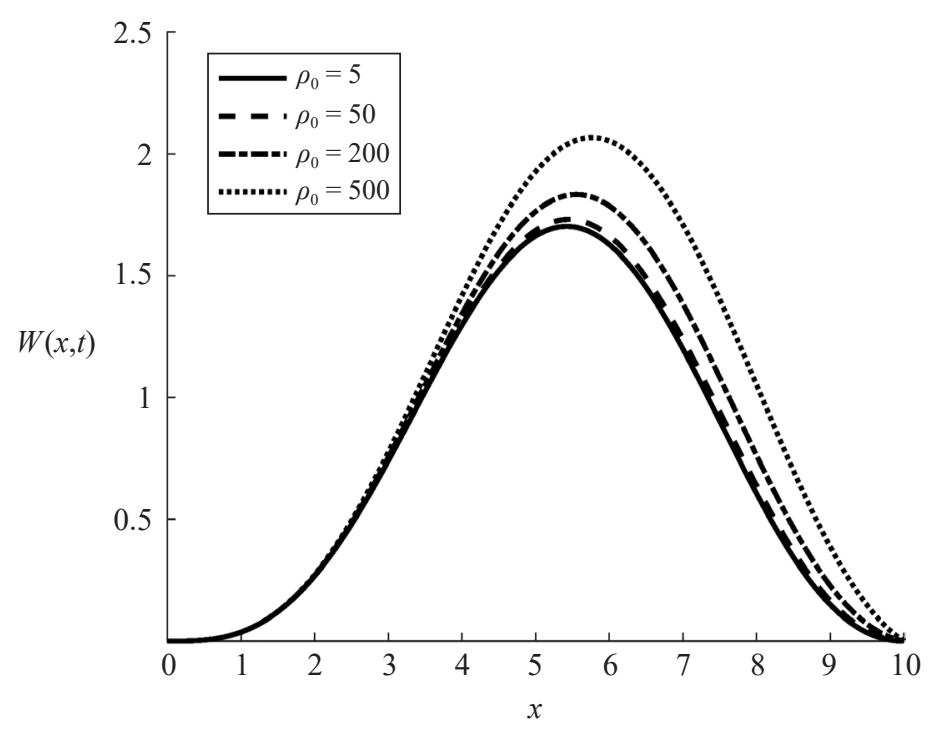

Figure 6(a): Deflection of beam for various values of $\rho_{0}$ (acceleration considered).

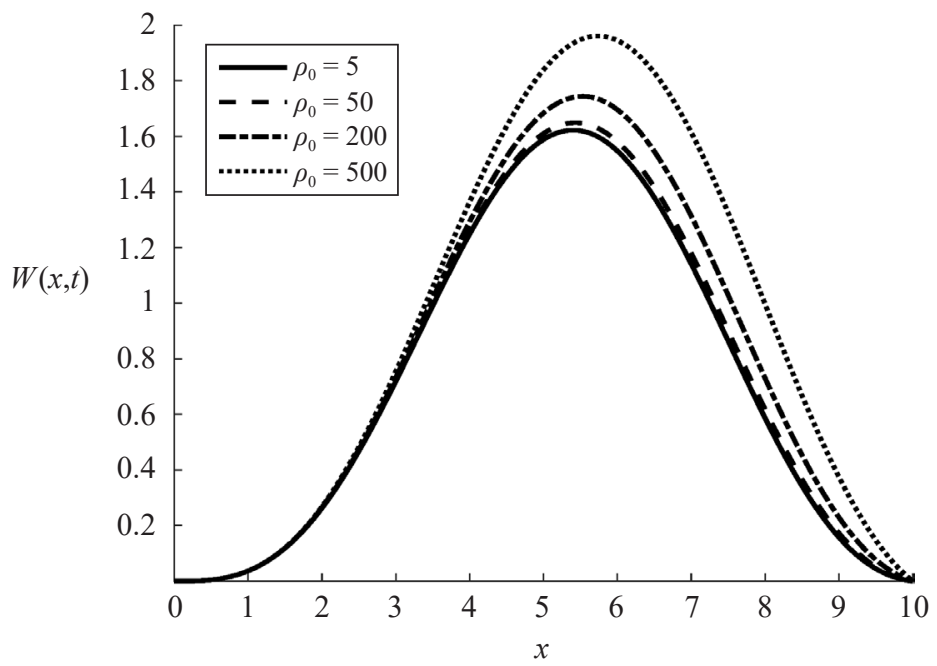

Figure 6(b): Deflection of beam for various values of $\rho_{0}$ (acceleration neglected). 


\section{CONCLUSION}

The dynamic behaviour of Euler-Bernoulli beam on Winkler foundation subjected to partially distributed moving load is investigated in this study. Analytical solutions are used to solve the problem under consideration. The beam responses investigated are deflection, shear force, velocity profile and bending moment. The beam responses are observed for different loads at different velocities. The analytical solutions are validated through comparison with existing analytical and numerical solutions. The series solution used here can be applied to any related analytical studies of this model. Acceleration of a traveling mass over a structural system, highly affects the dynamic response of the structural system. This will give the engineers some advantages to make a more realistic modelling of structural systems.

\section{REFERENCES}

1. Taha, M. (2014). Recursive differentiation method for boundary value problems: Application to analysis of a beam. J. Theor. Appl. Mech., Sofia, 44(2), 57-70, https://doi.org/10.2478/jtam-2014-0010.

2. Frýba, L. (1972). Vibration of solids and structures under moving loads. Groningen, Netherlands: Noordhoff International Publishing, https://doi.org/10.1007/978-94-011-9685-7.

3. Hayir, A. (2010). Dynamic behavior of an elastic beam on a Winkler foundation under a moving load. Paper presented at the International Conferences on Recent Advances on Geotechnical Earthquake Engineering and Soil Dynamics, San Diego, California, 24-29 May.

4. Gbadeyan J. A., Hammed F. A. \& Titiloye E. O. (2005). Dynamic behavior of viscoelastically connected beams carrying uniform partially distributed moving masses. NJPAS, 20, 1891-1905.

5. Usman, M. A. \& Hammed, F. A. (2007). Dynamic response of a Bernoullibeam with viscous and structural damping coefficients subjected to partially distributed moving load. The Journal of the Mathematical Association of Nigeria, 34(2B), 452-461.

6. Ratnadeep, P. (2015). Dynamic behaviour of a cantilever beam subjected to moving mass and elastic end constraint. Int. J. Innov. Res. Sci. Eng. Technol., 4(7), 6241-6246.

7. Usman, M. A., Hammed, F. A. \& Onitilo, S. A. (2015). Vibration of Timoshenko beam subjected to partially distributed moving load. J. Sci. Res. Stud., 2(4), 80-86. 
8. Oni, S. T. \& Ayankop-Andi, E. (2017). On the response of a simply supported non-uniform Rayleigh beam to traveling distributed loads. J. Nigerian Math. Soc., 36(2), 435-457.

9. Oni, S. T. \& Jimoh, A. (2016). Dynamic response to moving concentrated loads of simply supported pre-stressed Bernoulli-Euler beam resting on bi-parametric subgrades. Int. J. Sci. Eng. Res., 7(9), 584-600.

10. Fatemeh, S. \& Elipakci, H. R. (2018). Analytical solution for modal analysis of Euler-Bernoulli and Timoshenko beam with an arbitrary varying cross-section. Mathematical Models in Engineering, 4(3), 164174, https://doi.org/10.21595/mme.2018.20116.

11. Jimoh, S. A., Ogunbamike, O. K. \& Olanipekun, A. O. (2018). Dynamic response of non-uniformly prestressed thick beam under distributed moving load travelling at varying velocity. Asian Res. J. Math., 9(4), 1-18, https://doi.org/10.9734/ARJOM/2018/41327.

12. Pasterer, C. A., Tan, C. A. \& Bergman, L. A. (2001). A new method for calculating bending moment and shear force in moving load problems. J. Appl. Mech., 68(2), 252-259, https://doi.org/10.1115/1.1356028. 